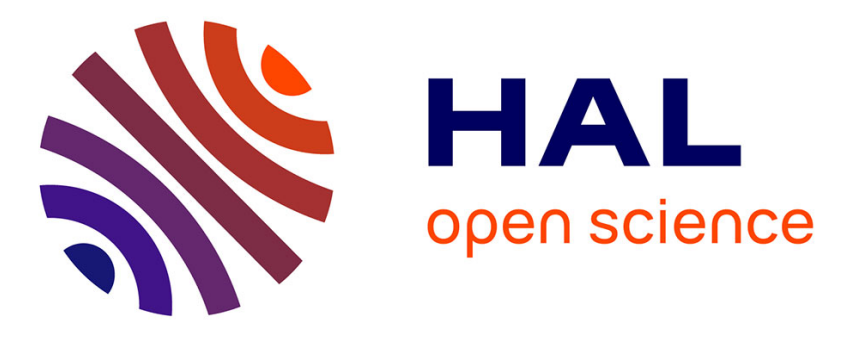

\title{
Dynamic covalent conjugated polymer epitaxy on graphene
}

\author{
Lea Assies, Chaoying Fu, Petr Kovaříček, Zdeněk Bastl, Karolina A. \\ Drogowska, Jan Lang, Valentino L. P. Guerra, Paolo Samori, Emanuele Orgiu, \\ Dmitrii F. Perepichka, et al.
}

\section{To cite this version:}

Lea Assies, Chaoying Fu, Petr Kovaříček, Zdeněk Bastl, Karolina A. Drogowska, et al.. Dynamic covalent conjugated polymer epitaxy on graphene. Journal of Materials Chemistry C, 2019, 7 (39), pp.12240-12247. 10.1039/C9TC03155C . hal-03017539

\section{HAL Id: hal-03017539 \\ https://hal.science/hal-03017539}

Submitted on 20 Nov 2020

HAL is a multi-disciplinary open access archive for the deposit and dissemination of scientific research documents, whether they are published or not. The documents may come from teaching and research institutions in France or abroad, or from public or private research centers.
L'archive ouverte pluridisciplinaire HAL, est destinée au dépôt et à la diffusion de documents scientifiques de niveau recherche, publiés ou non, émanant des établissements d'enseignement et de recherche français ou étrangers, des laboratoires publics ou privés. 


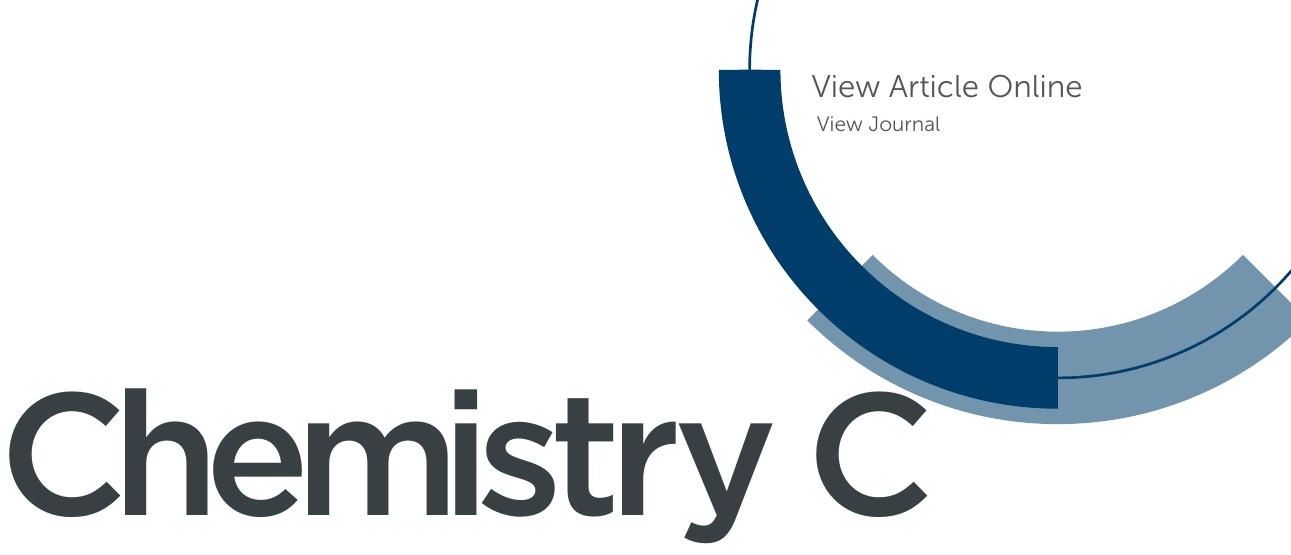

Materials for optical, magnetic and electronic devices

\section{Accepted Manuscript}

This article can be cited before page numbers have been issued, to do this please use: L. Assies, C. Fu, P. Kovaricek, Z. Bastl, K. Drogowska, J. Lang, V. L. P. Guerra, P. Samorì, E. Orgiu, D. Perepichka and M. Kalbac, J. Mater. Chem. C, 2019, DOI: 10.1039/C9TC03155C.
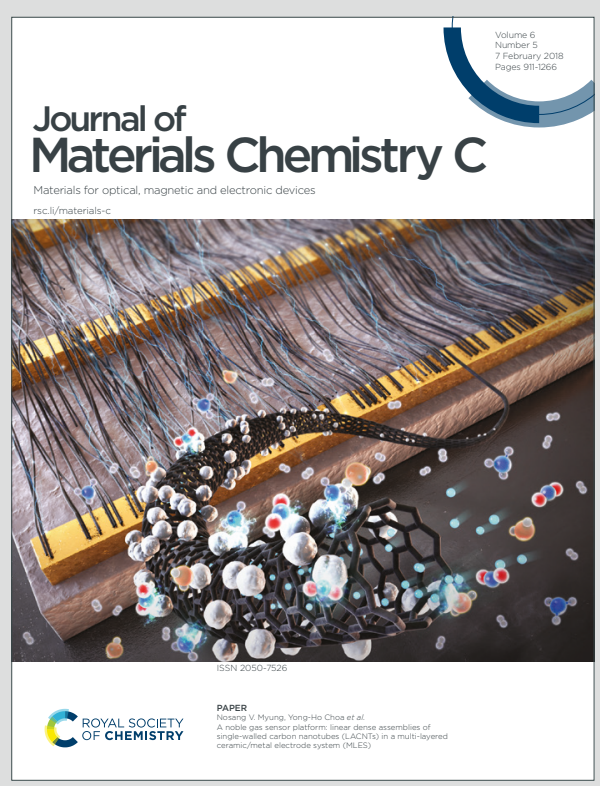

This is an Accepted Manuscript, which has been through the Royal Society of Chemistry peer review process and has been accepted for publication.

Accepted Manuscripts are published online shortly after acceptance, before technical editing, formatting and proof reading. Using this free service, authors can make their results available to the community, in citable form, before we publish the edited article. We will replace this Accepted Manuscript with the edited and formatted Advance Article as soon as it is available.

You can find more information about Accepted Manuscripts in the Information for Authors.

Please note that technical editing may introduce minor changes to the text and/or graphics, which may alter content. The journal's standard Terms \& Conditions and the Ethical guidelines still apply. In no event shall the Royal Society of Chemistry be held responsible for any errors or omissions in this Accepted Manuscript or any consequences arising from the use of any information it contains. 


\section{Dynamic covalent conjugated polymer ${ }^{\text {sisisc }}$ epitaxy on graphene}

Lea Assies, ${ }^{1,2} \S$ Chaoying Fu, ${ }^{3,4,5} \S$ Petr Kovaříček, ${ }^{1,}{ }^{*}$ Zdeněk Bastl, ${ }^{1}$ Karolina A. Drogowska, ${ }^{1}$ Jan Lang, ${ }^{6}$ Valentino L. P. Guerra, ${ }^{1}$ Paolo Samorì, ${ }^{3}$ Emanuele Orgiu, ${ }^{5,}{ }^{*}$ Dmitrii F. Perepichka, ${ }^{4,}{ }^{*}$ Martin Kalbác ${ }^{1,{ }^{*}}$

${ }^{1}$ J. Heyrovsky Institute of Physical Chemistry of the Czech Academy of Sciences, Dolejškova 2155/3, 18223 Prague, Czech Republic

${ }^{2}$ Department of Chemistry \& IRIS Adlershof, Humboldt-Universität zu Berlin, Brook-Taylor Str. 2, 12 489 Berlin, Germany

${ }^{3}$ University of Strasbourg, CNRS, ISIS UMR 7006, 8 allée Gaspard Monge, F-67000 Strasbourg, France

${ }^{4}$ Department of Chemistry, McGill University, 801 Sherbrooke Street W., Montreal, QC, Canada H3A OB8

${ }^{5}$ INRS, Énergie Matériaux Télécommunications Centre, 1650 boulevard Lionel-Boulet, Varennes (Québec) J3X 1S2, Canada

${ }^{6}$ Department of Low Temperature Physics, Faculty of Mathematics and Physics, Charles University, V Holešovičkách 2, 18000 Praha 8, Czech Republic

* martin.kalbac@jh-inst.cas.cz *emanuele.orgiu@emt.inrs.ca *dmitrii.perepichka@mcgill.ca *petr.kovaricek@jh-isnt.cas.cz

$\S$ These authors equally contributed to the work

\section{Abstract}

Hybrid heterostructures formed from ordered molecular layers on bidimensional materials can have unique properties differing from those of their bulk phases. By employing principles of dynamic covalent chemistry, we have synthesized a series of novel conjugated polyimines that form epitaxial ordered monolayers on graphene. The interplay between molecular physisorption and dynamic polymerization at the solid-liquid interface drives the formation of longer chains at the surface with higher rates than in solution. The physico-chemical properties of such assemblies at different length scales on graphene were investigated by a combination of experimental techniques. 'Covalent dynamic epitaxy' was also found to modulate the properties of both substrate and dynamers such as doping and photoluminescence, respectively.

\section{Introduction}

Mastering the mutual interactions at the interface between molecules and two-dimensional (2-D) materials holds great potential to generate novel hybrid 2-D materials displaying a desired set of physical properties. ${ }^{1-5}$ Among 2-D materials, graphene is a well-known platform possessing atomic flatness combined with exceptional opto-electronic characteristics, ${ }^{6-9}$ high transparency and mechanical robustness. ${ }^{10-13}$ It has been well documented that molecules can form crystalline epitaxial architectures on the basal plane of highly oriented pyrolytic graphite (HOPG). ${ }^{14-19}$ In addition, supramolecular epitaxial assemblies can form on individual graphene sheets, and the resulting hybrid heterostructures exhibit atomically precise and periodic patterns that can generate 
superlattices..$^{20}$ It has been shown that employing van der Waals interactions between grapheneedrindcle Online supramolecular networks is a powerful strategy to modulate the properties of graphene without perturbing its pristine $\mathrm{sp}^{2}$ honeycomb lattice, ${ }^{21-23}$ the common drawback of covalent functionalization. ${ }^{24-26}$ On the other hand, weak supramolecular interactions impose different challenges such as lower stability of the lattice, lattice mismatch between graphene and the epitaxial layer, or lattice defects, both in the substrate and the ad layer. All of these factors can be detrimental for the overall properties of the hybrid, and consequently the performance of the final device when such hybrid structures are employed as the active layer. ${ }^{27,28}$

Here we use principles of dynamic covalent chemistry ${ }^{29}$ (DCC) to achieve on-surface synthesis of dynamic conjugated polymers - dynamers. ${ }^{30-34}$ When occurring at a solid-liquid interface, dynamic covalent chemistry comprises reversible interactions at both covalent and non-covalent levels. Noncovalent interactions exist between the molecular adlayer and substrate surface. ${ }^{20,35}$ Covalent tethering occurs between ad hoc functionalized molecular building blocks which interact through reversible covalent bonds, thereby improving the robustness of the assembly. ${ }^{36-38}$ The reversible nature of the bond formation allowed by DCC enables thermodynamic control and results in highly ordered self-assembled structures. ${ }^{16,20,29,35,39}$ These features make dynamic bonds ideally suited for fabrication of surface-confined architectures including polymers and covalent organic frameworks. ${ }^{22,35,40-47}$ The concept of thermodynamically controlled supramolecular dynamer epitaxy is particularly attractive in the context of conjugated polymers, largely employed as (opto)electronic materials. ${ }^{48-51}$ In our work, we employed novel bifunctional aromatic aldehydes decorated with long alkyl chains to induce self-assembly on $s p^{2}$-carbon surfaces (monolayer graphene and HOPG), and copolymerized them with diamines forming polyimines. The monomers were designed to provide a different degree of conformational flexibility and $\pi$-conjugation. In particular, we investigated (i) how the structural information encoded in the molecule's constitution influences the final 2-D dynamic covalent assembly; (ii) how the spectroscopic properties of the final polymer are affected by epitaxial adsorption on graphene and how this adsorption affects the properties of the graphene substrate. The combined results, gathered with a broad arsenal of spectroscopic and microscopy techniques, provide new insights into the dynamic molecule/graphene interface.

\section{Results}

\subsection{Molecular design and synthesis}

To explore the dynamic covalent polymerization on graphene, we focused on the prototypical case of imine chemistry, by exploiting aldehyde and diamine building blocks. Three aromatic dialdehydes were synthesized from hydroquinone (HQ), resorcinol (RC) and 2,2'-biphenol (BP) precursors via $O$ alkylation with $n$-octadecylbromide followed by bromination with NBS, lithiation with $n$-BuLi and formylation by $\mathrm{N}, \mathrm{N}$-dimethylformamide (see SI Section 1 ). We anticipated that the position at which the aliphatic chains are tethered to the aromatic core would result in different confinement criteria for the polymer self-assembly and, thus, that the information contained in the constitution of each of these monomers would translate into the polymer self-assembly in 2-D space. We chose the following three diamine counterparts: hydrazine (hd, which supposedly forms the tightest packed 2$D$ layers), $p$-phenylenediamine (pp, which provides $\pi$-conjugation and rigidity but expands the lattice packing), and ethylenediamine (eda, which acts as a flexible non-conjugating linker). The overview of the employed structures and their acronyms is provided in Figure 1. 


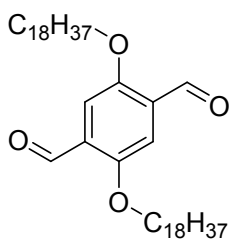

HQ

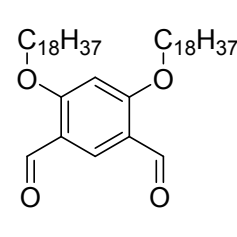

RC

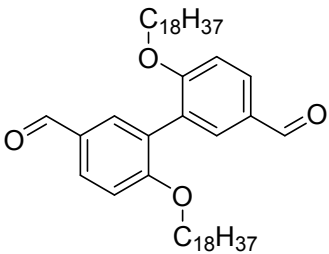

BP<smiles>NCCN</smiles><smiles>Nc1ccc(N)cc1</smiles>

pp
$\mathrm{H}_{2} \mathrm{~N}-\mathrm{NH}_{2}$

hd

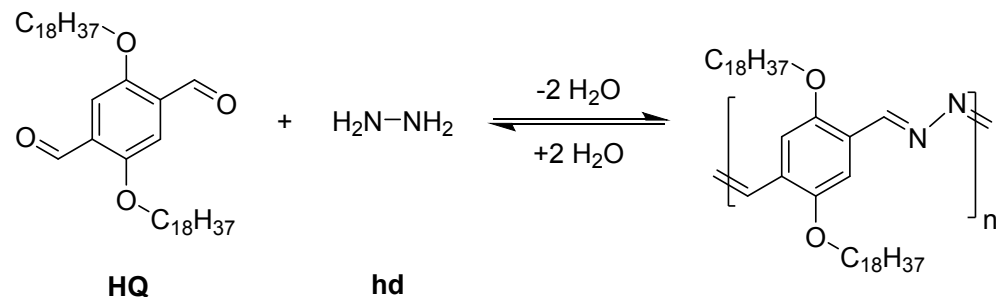

HQ:hd

Figure 1 Structures and their acronyms used in the study. Dialdehyde derivatives ( $H Q, R C, B P$ ) react on both carbonyl groups with diamines (eda, pp, hd) to give a total of nine corresponding polyimines. The polymer identifier is composed of the acronyms of its components, for example of HQ:hd.

\subsection{Dynamic polymerization process in solution}

The polymerization reactions were probed at high concentration $(20 \mathrm{mM})$ by NMR spectroscopy and at low concentration $(10 \mu \mathrm{M})$ by UV-Vis spectroscopy. While in NMR experiments the reaction occurs without catalytic assistance over several days, the reaction kinetics as monitored by UV-Vis spectroscopy is too slow to be followed experimentally. As reported earlier, the reaction can be catalyzed by acids or amines, ${ }^{37,52}$ which was also confirmed in our experiments by addition of octanoic acid or $n$-propylamine in UV-Vis and NMR experiments, respectively (see SI). The polymers were also synthesized in bulk by mixing the two constituents in a $1: 1$ ratio and reacting at $60^{\circ} \mathrm{C}$ for three days.

The degree of polymerization of HQ:hd was determined by gel permeation chromatography (GPC) and diffusion measurement by NMR spectroscopy. The GPC chromatogram shows a relatively broad peak with several maxima corresponding to fractionation and re-equilibration of the dynamer on the column. Molecular weight distribution analysis (see SI Section 8) revealed a dominant mass at around $5 \mathrm{kDa}$, corresponding approximately to a 7-mer and the smaller peaks correspond to lower oligomers down to the monomer. This result was corroborated using ${ }^{1} \mathrm{H}$ pulsed field gradient NMR spectroscopy, which estimated a chain size of $n=8 \pm 2$ (details in SI Section 7). This estimation is in a good agreement with the values obtained from GPC.

The thermodynamic stability of the $\mathrm{C}=\mathrm{N}$ bond differs for different imine derivatives. Under the conditions employed, the aromatic amines are less stable and prone to exchange by aliphatic amines, which can again be exchanged by hydrazine derivatives. ${ }^{37}$ However, in the present case, the conjugation extension favours pp incorporation into the polymer formed from eda giving approximately statistical product ratio. Yet, upon addition of hd both diamines are expelled from the 


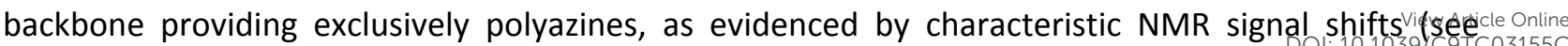
Figure 2a).
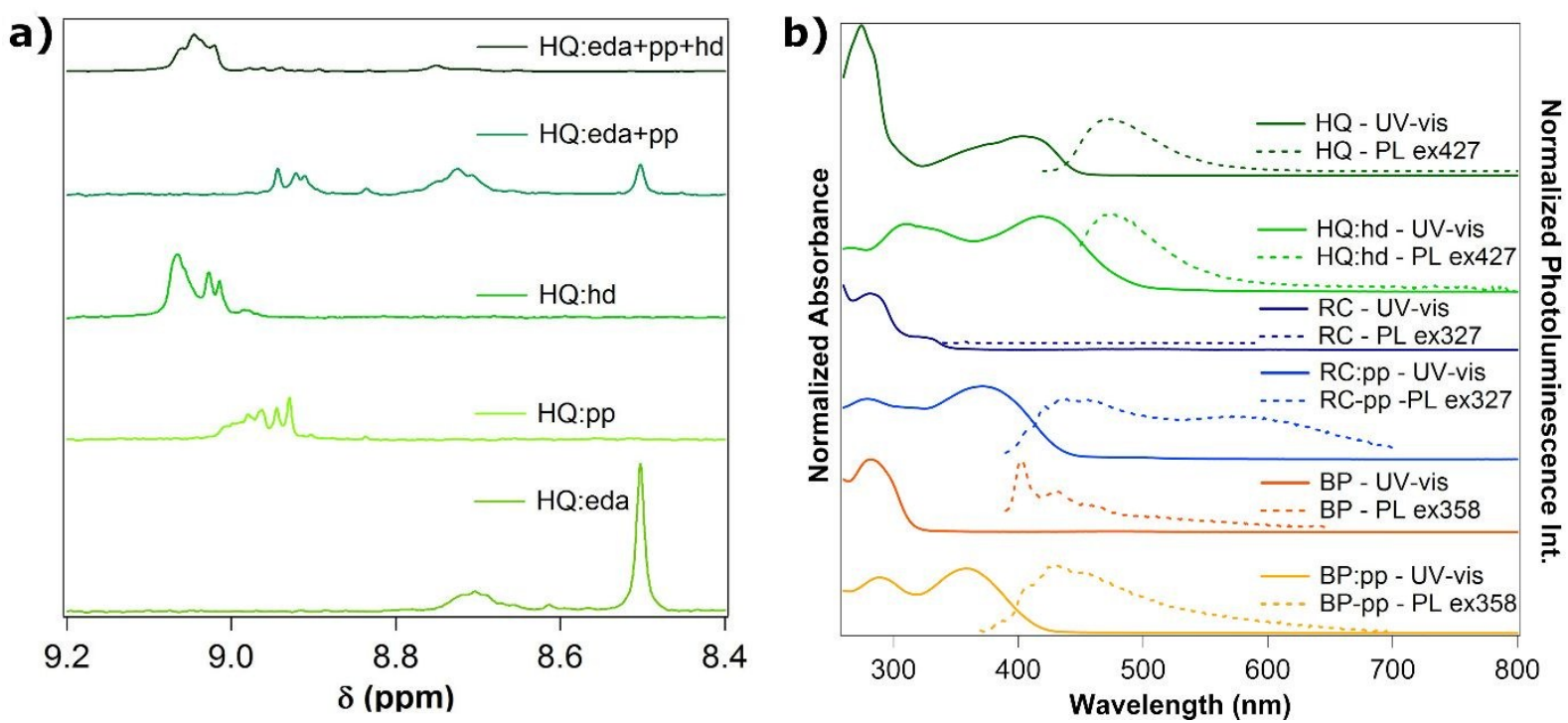

Figure 2 a) Azomethine proton region of the NMR spectra of $H Q$ condensed with different diamines and their mixtures. The polymers with eda shows two signals at around 8.5 and 8.7 ppm, with pp at around 8.9-9.0 and that derived from hd at 9.09.1. When eda and pp are put in competition, formation of both products is observed, while addition of hd gives exclusively polyazine chains. All spectra are recorded in equilibrium and presence of several signals in each spectrum is due to the dynamic network of polymers in the mixtures. b) Selection of UV-Vis and PL spectra of the starting dialdehyde monomers and the corresponding polymers formed with conjugating diamines linkers pp and hd. The absorption and emission is redshifted due to conjugation extension upon imine formation. Spectra of other polymer can be found in the SI.

UV-Vis spectra of polymers formed in solution from pp or hd show an absorption shift towards lower energies indicating extension of the conjugation length by polyimine formation (see Figure $2 b$ and individual traces in the $\mathrm{SI}$ ). This is further evidenced by photoluminescence (PL) spectra in which the polymerization leads to a red-shifted emission maxima and significant broadening of the band supporting conjugation extension, which is indicative of a dynamic network of polymer chains with various length (Figure $2 \mathrm{~b}$ ). It is important to point out that PL measurements carried out in solution only reflect the effective conjugation length which is shorter than the actual polymer backbone due to conformational disorder. Thus, the emission is red-shifted when the polymer is planarized on the surface. For instance, the emission maximum for the HQ monomer occurs at $473 \mathrm{~nm}$, for HQ:hd in solution at $475 \mathrm{~nm}$ and in a drop-casted film at $550 \mathrm{~nm}$ (SI Figure S4), but when measured as a $10 \mathrm{~nm}$ film on graphene the emission shifts to $624 \mathrm{~nm}$ (Figure 3), i.e. by $0.62 \mathrm{eV}$ with respect to the solution.

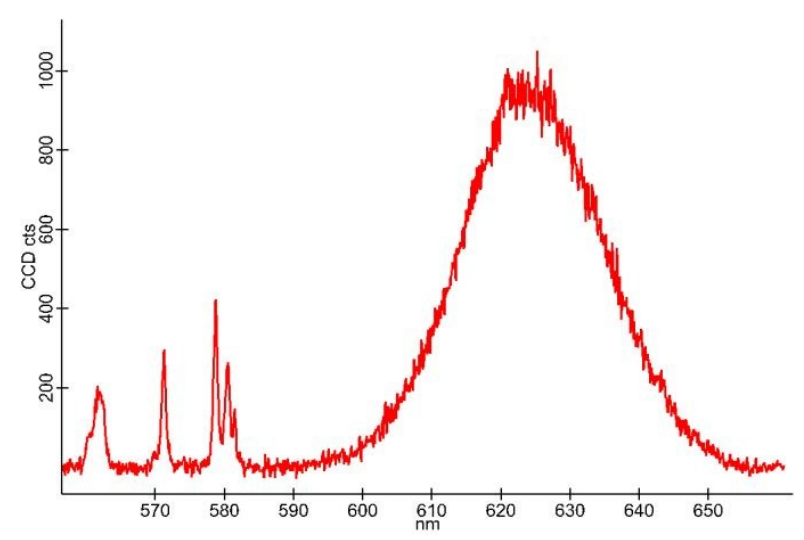

Figure 3 Photoluminescence spectrum of HQ:hd measured with confocal Raman spectrometer (excitation wavelength 532 $\mathrm{nm}$ ) on a $10 \mathrm{~nm}$ thin film on graphene. Strong red shift of the emission is observed to about $624 \mathrm{~nm}$ due to planarization of the polymer backbone on the surface. 


\subsection{Raman spectroscopy of hybrid heterostructures}

Raman spectroscopy is a non-invasive method to investigate solid materials. It is extensively used to determine strain and doping levels in graphene and related 2-D materials, ${ }^{53}$ and was applied for our epitaxial layers to study the formation of the polymers and their interactions with graphene. Graphene was grown by the chemical vapour deposition method (CVD) on a copper foil, and $1 \mathrm{~cm} \times 1$ $\mathrm{cm}$ sheets were transferred onto $\mathrm{Si} / \mathrm{SiO}_{2}$ substrates by the $\mathrm{Cu}$ etching/polymer assisted method as described earlier (see SI Section 2).54,55 The Raman spectrum of the so-prepared samples exhibited only $G$ and $2 D$ modes at around 1600 and $2700 \mathrm{~cm}^{-1}$, respectively, without any detectable $D$ mode (Figure 4a). The PL observed in solution and in thick films is efficiently quenched when the films were deposited on graphene from low-concentration solutions, ${ }^{56}$ and the Raman spectra clearly show the bands corresponding to both the polymers and graphene (Figure 4a). The strain-doping analysis, calculated from the $2 \mathrm{D}$ and $\mathrm{G}$ band positions, ${ }^{53}$ was performed on a pristine graphene and after deposition of the dynamer. From the analysis, we find that, while the strain is negligible, the presence of the HQ:hd polymer leads to a decrease in graphene's pristine doping from $1.35 \times 10^{13}$ to $9.66 \times 10^{12} \mathrm{~cm}^{-2}$, i.e. by about $30 \%$ (see SI Section 4.4).

HQ:hd bands are found at $1293 \mathrm{~cm}^{-1}, 1525 \mathrm{~cm}^{-1}$, and $1573 \mathrm{~cm}^{-1}$ corresponding to the $-\left(\mathrm{CH}_{2}\right)_{\mathrm{n}}-$ twisting vibration, the symmetric $\mathrm{C}=\mathrm{N}-\mathrm{N}=\mathrm{C}$ stretch of aryl azines, and the $\mathrm{C}=\mathrm{C}$ stretching vibration of the benzene rings, respectively. ${ }^{57}$ The Raman shift and shape of the $\mathrm{CH}_{2}$ twisting band are useful probes into the self-assembly of the aliphatic chains ${ }^{58,59}$ - a sharp band closer to $1293 \mathrm{~cm}^{-1}$ indicates high crystallinity while a shift above $1300 \mathrm{~cm}^{-1}$ and band broadening is typical for the amorphous phase. The polymer Raman scattering is most efficient in the excitation range of $458-648 \mathrm{~nm}$, presumably due to resonance enhancement. The experimental traces for other polymers with corresponding discussion is provided in the SI Figure S5-6. Raman mapping was used to study if the polymer assembles selectively on graphene vs. the bare substrate. The obtained spectra were fitted and (co)localization of the polymer and graphene bands together with optical image was investigated (SI Table 3). A selective film formation on graphene was observed for the HQ- and RC-based polymers upon varying the concentration of the spin-coated solution, but the BP polymers showed poor selectivity, even in highly diluted $(\leqslant n M)$ solutions of monomers. Excess of spin-coated material can be rinsed with chloroform, but rinsing with solvent does not lead to complete film removal in any case.
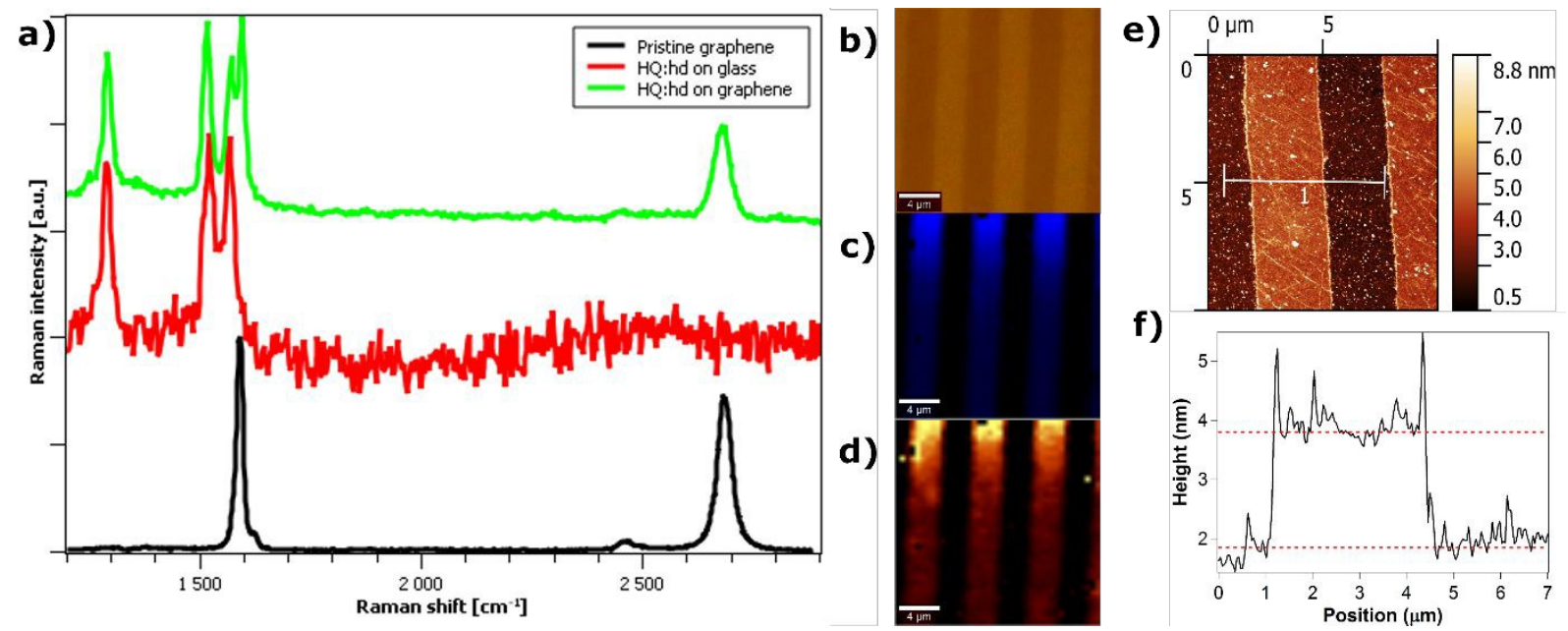

Figure 4 a) Raman spectrum of the HQ:hd polymer film on glass and spin-coated on graphene on a $\mathrm{Si}_{\mathrm{SiO}} \mathrm{support}_{\text {. The }}$ characteristic polymer bands are clearly visible as well as the graphene $G$ and $2 D$ modes at $1600 \mathrm{~cm}^{-1}$ and $2693 \mathrm{~cm}^{-1}$, respectively. b) Optical image of patterned graphene stripes with an on-surface grown polymer layer (scale bar equals 4 $\mu \mathrm{m})$. Maps of the intensity of graphene's $2 D$ mode (c) and the polymer band at $1293 \mathrm{~cm}^{-1}$ (d) are fully colocalized and match 
the optical image (scale bars equal $4 \mu \mathrm{m}$ ). e) AFM image acquired over the graphene stripe with height profile (f) indictentingicle Online an average graphene-polymer (on-surface grown at $1 \mu \mathrm{M}$ monomer concentration) hybrid layer thickness of abbu1t. $203 \mathrm{~nm}$. C9TC $03155 \mathrm{C}$

To study the on-surface growth of $\mathrm{HQ}:$ hd on graphene, we have immersed graphene sheets on $\mathrm{Si} / \mathrm{SiO}_{2}$ substrates into $\mathrm{HQ}$ solution of concentration from $1 \mathrm{mM}$ down to $1 \mathrm{pM}$. After a 30 minute equilibration period, one equivalent of hd was added and the reaction was kept at room temperature for 12 hours. Successful polymer film formation was observed at all concentrations as confirmed by Raman mapping showing co-localization of the graphene 2D mode and a polymer band at $1293 \mathrm{~cm}^{-1}$ (Figure 4b-d and SI section 9.2). Using atomic force microscopy, the layer thickness (at graphene edges or defects) was measured as $10 \mathrm{~nm}$ at $1 \mathrm{mM}$ concentration down to about $2 \mathrm{~nm}$ at 1 pM level, i.e. close to monolayer coverage (see SI for experimental traces). By measuring the height profile over HQ:hd self-assembled layers from a $1 \mu \mathrm{M}$ solution on 3- $\mu \mathrm{m}$ wide graphene stripes, an average hybrid composite thickness of about $2 \mathrm{~nm}$ was determined (see Figure 4 e-f). Spin-coating of polymer solutions $(0.3 \mathrm{mg} / \mathrm{mL})$ in chloroform provided homogenous $\sim 5 \mathrm{~nm}$ thick layers. Raman spectra of all HQ:hd films showed a sharp band at $1293 \mathrm{~cm}^{-1}$, thus confirming high crystallinity even in thicker films, in contrast to other polymers which are more disordered (SI Figure S6b).

The surface has a 'catalytic' effect, dramatically accelerating polymerization. ${ }^{60,61}$ Extrapolating from the second-order kinetics measured at a concentration of $20 \mathrm{mM}$ (SI Figure S22), the reaction in $1 \mathrm{nM}$ solution would require more than $10^{7}$ days to reach equilibrium. However, dynamic self-assembly on graphene is successfully achieved in less than 12 hours. At $1 \mathrm{nM}$ concentration, the polymer layer self-assembly appears continuous by Raman mapping, even though most of the molecules present in the solution must assemble on the surface to form a continuous layer (approx. $1 \mathrm{~cm}^{2}, 25 \mathrm{~mL}$ of $1 \mathrm{nM}$ solution). At $1 \mathrm{pM}$ concentration, the polymer Raman bands are not homogeneously mapped over the whole graphene area.

\subsection{Surface-templated polymerization}

Scanning Tunnelling Microscopy (STM) is a powerful characterization technique capable of imaging surface adsorbed molecules with sub-molecular resolution. ${ }^{62-64}$ We used STM to study the selfassembly and on-surface polymerization yielding macromolecules with controlled shape dictated by the constitution of the starting building blocks. The long octadecyl side-chains in HQ-monomers provide not only good solubility in organic solvents, but also a strong physisorption enthalpy on the $s p^{2}$ carbon surface. The adsorption energy of a benzene ring on graphite surface is $0.5 \pm 0.08 \mathrm{eV}, 65$ which is only one fifth of the adsorption energy of two fully extended octadecyl side-chains on graphite surface $\left(\sim 2 \times 1.16 \mathrm{eV}, \sim 64.2 \mathrm{meV}\right.$ per $\left.\mathrm{CH}_{2}\right) .{ }^{66}$ The molecular assembly is further stabilized via interdigitation of neighbouring chain lamellar structures. The use of Schiff-base polymerization enables dynamic exchange of the imine constituents at the solid-liquid interface. ${ }^{29}$

When applying a 1:1 molar ratio mixture of $\mathbf{H Q}$ and hd solution (0.1 $\mathrm{mM}$ each) in trichlorobenzene (TCB) and octanoic acid ( $0.1 \% \mathrm{v} / \mathrm{v}$ as an acid catalyst) onto an HOPG substrate, the immediate formation of lamellar structures is observed (Figure 5a). Because of the small size of $\mathbf{H Q}$ and hd monomers, the molecular dynamics at this concentration at room temperature is faster than the STM imaging, and thus, they could not be visualized (Figure S16 and S17). Therefore, the appearance of lamellar architectures indicate the in situ formation of HQ:hd polymers on HOPG, which was also confirmed by UV-Vis measurement of the same solution mixture and by XPS spectroscopy of the film (Figure S13-S15). The area of the long-range ordered single domains often exceeds $100 \times 100 \mathrm{~nm}^{2}$ and the lamellae are up to $\sim 150 \mathrm{~nm}$ long. However atomic resolution could not be achieved to confirm the presence of single or multiple polymer backbones assembled within each lamella. 


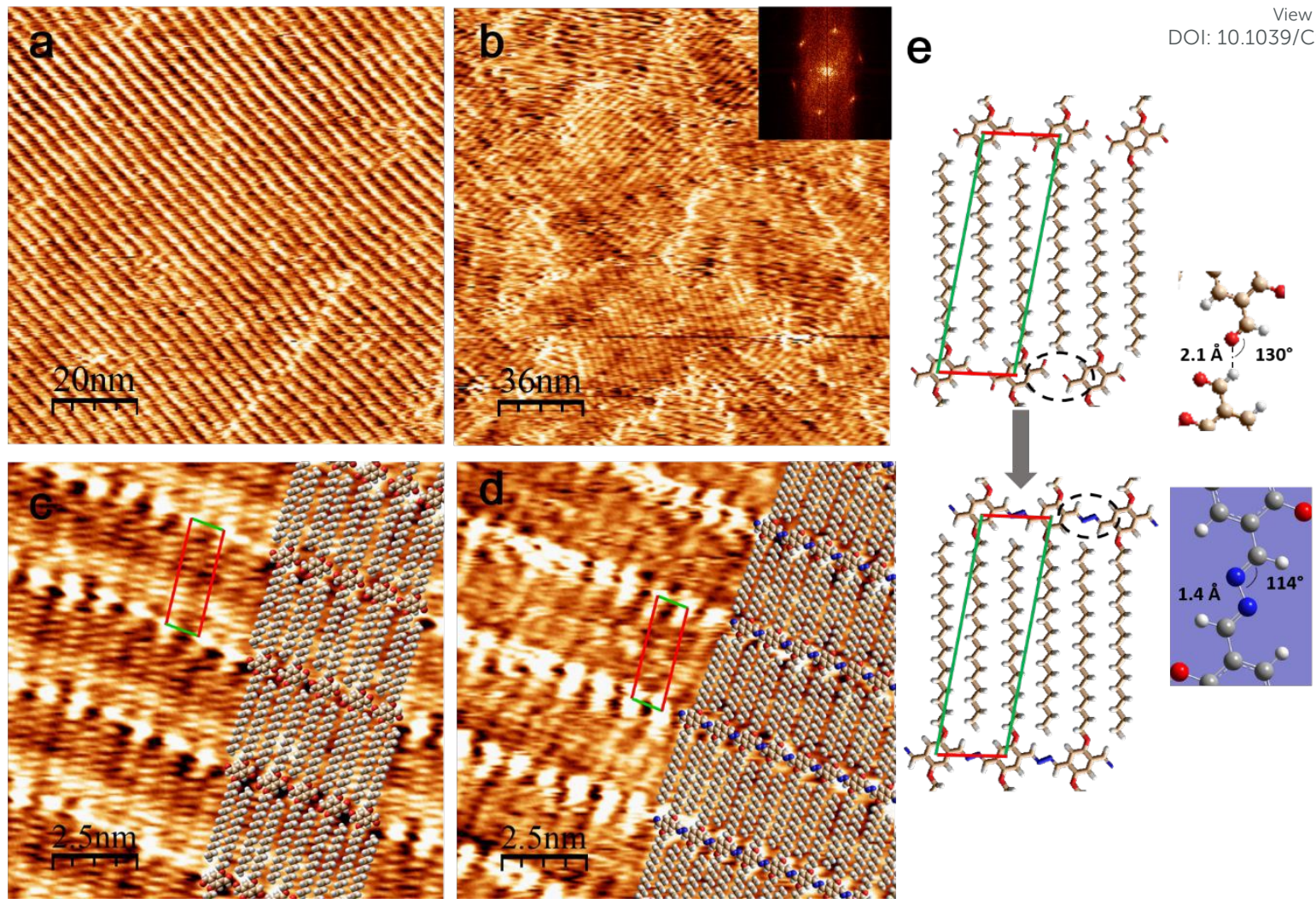

Figure 5 a) STM image of in-situ polymerization of HQ and hd at the TCB/HOPG interface showing single domain lamellar structures extending $100 \mathrm{~nm}$ in two dimensions. b) Self-assembly of bulk-synthesized HQ:hd polymers at the TCB/HOPG interface. c) High resolution STM image of HQ self-assembly in $1 \mathrm{mM}$ solution at TCB/HOPG interface. The STM image is calibrated with the underlying HOPG lattice, showing a unit cell dimension of $a=1.0 \pm 0.1 \mathrm{~nm}, b=3.0 \pm 0.1 \mathrm{~nm}$ and $\alpha=81 \pm$ $1^{\circ}$. d) High resolution STM image of in-situ grown HQ:hd polymer in $0.1 \mathrm{mM}$ monomer concentration solution at TCB/HOPG interface. The STM image is calibrated with the underlying HOPG lattice, showing a unit cell dimension of $a=0.9 \pm 0.1 \mathrm{~nm}, b$ $=2.9 \pm 0.1 \mathrm{~nm}$ and $\alpha=82 \pm 1^{\circ}$. e) Molecular modelling shows a topographic similarity between $\mathrm{HQ}$ and $\mathrm{HQ}: \mathrm{hd}$ polymer lamellae facilitating the on-surface reaction by monomer pre-assembly. The polymer backbone of HQ:hd was optimized by DFT calculation at B3LYP/6-31G(d) under periodic boundary conditions.

In comparison with in situ surface-grown polymers, we also imaged ex situ (i.e. in the flask) synthesized HQ:hd polymer drop-casted $(0.07 \mathrm{mg} / \mathrm{mL}$ in TCB) onto HOPG. In contrast to the extended domains formed during in-situ polymer growth, the solution-synthesized polymer aggregates into much smaller domains on HOPG, with an average area of $30 \times 30 \mathrm{~nm}^{2}$ (Figure $5 \mathrm{~b}$ ) and $120^{\circ}$ mutual orientations respecting the three-fold symmetry of the underlying HOPG (confirmed by 2D-FFT in Figure $5 b$ onset).

High-resolution STM images and molecular modelling indicate very similar unit cell dimensions of the HQ:hd polymer $\left(a=0.9 \pm 0.1 \mathrm{~nm}, \mathrm{~b}=2.9 \pm 0.1 \mathrm{~nm}, \alpha=82 \pm 1^{\circ}\right)$ and HQ monomer $(\mathrm{a}=1.0 \pm 0.1 \mathrm{~nm}, \mathrm{~b}$ $=3.0 \pm 0.1 \mathrm{~nm}, \alpha=81 \pm 1^{\circ}$ ) in the lamellar structures (Figure $5 \mathrm{c}-\mathrm{e}$ ). The molecular modelling also reveals that both the length and angle of the formed $\mathrm{C}=\mathrm{N}-\mathrm{N}=\mathrm{C}$ linkage are close to those of the $\mathrm{C}=\mathrm{O} \cdots \mathrm{H}-\mathrm{C}(=\mathrm{O})$ hydrogen-bonded assembly of $\mathrm{HQ}$ (Figure $5 \mathrm{e}$ ). These topographic similarities suggest that only limited molecular movements in the self-assembled $\mathbf{H Q}$ structure would be required for the formation of HQ:hd polymers, and thus the dynamic pre-assembly of HQ on HOPG might facilitate the long-range ordering of the in-situ grown HQ:hd polymers. This approach is reminiscent of topochemical polymerization of diacetylene derivatives on HOPG, in which the supramolecular ordering of diacetylene monomers favours polymerization within self-assembled monolayers. ${ }^{67-70} \mathrm{In}$ contrast, due to the lack of a topographic match between the monomer and polymer assemblies, lamellar structures observed for the in situ growth of other polymers based on $\mathbf{H Q}, \mathbf{R C}, \mathbf{h d}$ and $\mathbf{p p}$ 
monomers are much smaller than those of HQ:hd polymers (SI, Figure S18). In addition, BP-blistedcle Online polymers form no ordered assemblies at all. STM observations of the HQ:hd polymer grown on the CVD graphene showed that the lamellar structure formed is identical to that formed on HOPG (SI, Figure S20). However, the image quality is hampered by nano-rugae formed during graphene transfer. ${ }^{55,71}$

\section{Discussion}

The thermodynamically driven supramolecular dynamer epitaxy on graphene was investigated for a series of bifunctional aldehydes (HQ, RC, BP) and amines (hd, pp, eda), each combination providing a different dynamer. The polycondensation in solution was monitored by NMR and reached equilibrium in about 6 days The dynamic nature of the imine bond even when embedded in the backbone was confirmed by complete exchange of eda or pp from the polymer on addition of hd. The polymerization provides a dynamic network ${ }^{72-74}$ of $\mathrm{HQ}$ :hd polymers with average size of about 7-8 units, as determined by DOSY and GPC. In cases of hd and pp linkers, the polycondensation extends the conjugation and it can be evidenced spectroscopically by absorbance and PL maxima are red-shifted with respect to the monomer..$^{75,76}$

If the reaction occurs directly on a templating surface such as graphene or HOPG, the length of the polymer chains formed increases by more than an order of magnitude, as observed by STM and corroborated with PL measurements. PL is also very sensitive to molecular conformation rigidity. Thus, self-assembled films on graphene display a large PL red-shift $(0.62 \mathrm{eV})$ as compared to the polymer in solution. In addition to the thermodynamic effect, a dramatic kinetic enhancement of polycondensation was also observed on surfaces compared with in solution. We propose that in the dynamic polymerization process, the graphene surface acts as a template for monomer pre-assembly and thus facilitates the highly ordered surface-confined polymerization. The templating effect could also operate via establishing a high effective concentration of the reactive species at the surface. Furthermore, thanks to the fast imine dynamics and entropically preferred adsorption of larger species on the surface, ${ }^{29}$ the on-surface polymerization could compete with the adsorption of small agglomerates as in the case of in-solution synthesized polymers, thus rendering fully extended polymer networks.

On a molecular level, the polymers self-assemble into extended (>100 $\mathrm{nm}$ ) lamellar structures by aliphatic chain interdigitation, as evidenced by STM. This clearly shows that the substrate plays an important role in facilitating the high degree of polymerization of HQ:hd. Other dialdehydes do not form large crystalline domains on the surface, confirming that the constitutional design of molecules dictates the final self-assembly. The high crystalline order at a micron scale and larger can be conveniently followed by Raman mapping of the position and shape of the $1293 \mathrm{~cm}^{-1} \mathrm{CH}_{2}$ twisting band. In agreement with STM results, the behaviour of this twisting band shows that HQ:hd films have higher crystallinity than films of other polymers. Raman mapping can also be used to determine the spatial distribution of the deposited polymer (either on-surface grown or spin-coated) on graphene layers by colocalization of the characteristic polymer bands and graphene Raman modes. The preference for graphene substrate decreases with lower film crystallinity: HQ:hd has the highest selectivity, RC- and pp-based polymers show intermediate selectivity, and randomly packed BP-based dynamers display essentially no selectivity. This result suggests that feasible epitaxy of the dynamer layer on graphene has a large contribution to the selectivity of this layer. The polymer layer thickness, derived from AFM, can be varied between 2 to $10 \mathrm{~nm}$ by adjusting the monomer concentration during the on-surface polymerization. 
Spectral separation of the dynamer and graphene Raman signals also allows for the investigatibir of ofle Online both layers of the heterostructure simultaneously. Supported graphene is inherently doped by the substrate, which in our case is $\mathrm{SiO}_{2}$, leading to a rather high doping level $\left(>10^{13} \mathrm{~cm}^{-2}, \mathrm{p}\right.$-doping). After deposition of the dynamer on graphene, we observed a $30 \%$ decrease (approximately) in the doping level, and we propose that this is due to the compensation of the inherent doping by n-doping caused by the polymer layer.

\section{Conclusions}

In conclusion, the prepared hybrid molecular assemblies are confined to the $1 \times 1 \mathrm{~cm}^{2}$ graphene substrate with superb resolution and show highly ordered periodic arrangement at the nanoscale. The self-assembly process forms monocrystalline domains extending by areas exceeding $100 \times 100$ $\mathrm{nm}^{2}$. This stems from the interplay of two dynamic processes - adsorption/desorption of monomers on the surface and dynamic covalent polymerization forming polyimine chains. We have shown that the graphene and HOPG surfaces affects the dynamer formation and assembly, both kinetically and thermodynamically, and lead to periodic superstructures, with spectroscopic properties differing from the those observed in solutions containing these conjugated polymers. On the other hand, the assembly is also affecting its substrate by adjusting the doping level. Our results show that supramolecular conjugated dynamer epitaxy on carbon surfaces is an efficient strategy for bottomup fabrication of hybrid 2-D materials with tunable properties. This concept can be exploited in broad range of (opto)electronic devices such as semiconducting layers, sensors and (light-emitting) diodes. Mastering the underlying covalent dynamics at molecular level can generate hybrid molecule/2D interfaces that feature defect-healing and adaptation to a stimulus. As such, these hybrid interfaces could be exploited as their active layers in devices.

\section{Supporting information}

Electronic supplementary information (ESI) available: Synthetic protocols, additional spectra and images, polymer preparation and characterization, graphene synthesis and processing.

\section{Acknowledgements}

The work was supported by project No.s: 18-20357S and 18-09055Y (awarded by Czech Science foundation). The authors also acknowledge the assistance provided by the Research Infrastructures NanoEnviCz (Project No. LM2015073) supported by the Ministry of Education, Youth and Sports of the Czech Republic and the project Pro-NanoEnviCz (Reg. No. CZ.02.1.01/0.0/0.0/16_013/0001821) supported by the Ministry of Education, Youth and Sports of the Czech Republic and the European Union - European Structural and Investments Funds in the frame of Operational Programme Research Development and Education. E. O. and D. F. P. are supported by the Natural Sciences and Engineering Research Council of Canada (NSERC) through individual Discovery Grants. C.F. acknowledges the postdoctoral fellowship from Fonds de recherché du Québec - Nature et technologies (FQRNT). The NMR spectrometer at Charles University was purchased within the project CZ.2.16/3.1.00/21566 funded by the Operational Programme Prague - Competitiveness.

\section{References}

1 J. R. Potts, D. R. Dreyer, C. W. Bielawski and R. S. Ruoff, Polymer, 2011, 52, 5-25.

2 P. Solís-Fernández, M. Bissett and H. Ago, Chem. Soc. Rev., 2017, 46, 4572-4613.

3 V. Dhand, K. Y. Rhee, H. Ju Kim, D. Ho Jung, V. Dhand, K. Y. Rhee, H. Ju Kim and D. Ho Jung, J. Nanomater., 2013, 2013, e763953. 
4 F. Bonaccorso, L. Colombo, G. Yu, M. Stoller, V. Tozzini, A. C. Ferrari, R. S. Ruoff and V. Pellegriniticle Online Science, 2015, 347, 1246501.

5 T. Leydecker, M. Herder, E. Pavlica, G. Bratina, S. Hecht, E. Orgiu and P. Samorì, Nat. Nanotechnol., 2016, 11, nnano.2016.87.

6 K. Kostarelos and K. S. Novoselov, Nat. Nanotechnol., 2014, 9, 744-745.

7 B. H. Lee, J.-H. Lee, Y. H. Kahng, N. Kim, Y. J. Kim, J. Lee, T. Lee and K. Lee, Adv. Funct. Mater., 2014, 24, 1847-1856.

8 M. Sharon and M. Sharon, Graphene: An Introduction to the Fundamentals and Industrial Applications, John Wiley \& Sons, 2015.

9 A. K. Geim, Science, 2009, 324, 1530-1534.

10 K. S. Novoselov, A. K. Geim, S. V. Morozov, D. Jiang, Y. Zhang, S. V. Dubonos, I. V. Grigorieva and A. A. Firsov, Science, 2004, 306, 666-669.

11 K. S. Novoselov, A. K. Geim, S. V. Morozov, D. Jiang, M. I. Katsnelson, I. V. Grigorieva, S. V. Dubonos and A. A. Firsov, Nature, 2005, 438, 197-200.

12 A. K. Geim and K. S. Novoselov, Nat. Mater., 2007, 6, 183-191.

13 R. R. Nair, P. Blake, A. N. Grigorenko, K. S. Novoselov, T. J. Booth, T. Stauber, N. M. R. Peres and A. K. Geim, Science, 2008, 320, 1308-1308.

14 S. De Feyter and F. C. De Schryver, Chem. Soc. Rev., 2003, 32, 139-150.

15 S. De Feyter and F. C. De Schryver, J. Phys. Chem. B, 2005, 109, 4290-4302.

16 M. Gobbi, E. Orgiu and P. Samorì, Adv. Mater., 2018, 30, 1706103.

17 D. Guo, K. Sakamoto, K. Miki, S. Ikeda and K. Saiki, Phys. Rev. Lett., 2008, 101, 236103.

18 A. C. Hillier and M. D. Ward, Phys. Rev. B, 1996, 54, 14037-14051.

19 D. E. Hooks, T. Fritz and M. D. Ward, Adv. Mater., 2001, 13, 227-241.

20 M. Gobbi, S. Bonacchi, J. X. Lian, Y. Liu, X.-Y. Wang, M.-A. Stoeckel, M. A. Squillaci, G. D’Avino, A. Narita, K. Müllen, X. Feng, Y. Olivier, D. Beljonne, P. Samorì and E. Orgiu, Nat. Commun., 2017, 8, 14767.

21 Z. Li, H. V. Gorp, P. Walke, T. H. Phan, Y. Fujita, J. Greenwood, O. Ivasenko, K. Tahara, Y. Tobe, H. Uji-i, S. F. L. Mertens and S. D. Feyter, Nanoscale, 2017, 9, 5188-5193.

22 R. Phillipson, C. J. L. de la Rosa, J. Teyssandier, P. Walke, D. Waghray, Y. Fujita, J. Adisoejoso, K. S. Mali, I. Asselberghs, C. Huyghebaert, H. Uji-i, S. D. Gendt and S. D. Feyter, Nanoscale, 2016, 8, 20017-20026.

23 G. Gryn'ova and C. Corminboeuf, J. Phys. Chem. Lett., 2018, 2298-2304.

24 Z. Xia, F. Leonardi, M. Gobbi, Y. Liu, V. Bellani, A. Liscio, A. Kovtun, R. Li, X. Feng, E. Orgiu, P. Samorì, E. Treossi and V. Palermo, ACS Nano, 2016, 10, 7125-7134.

25 A. Criado, M. Melchionna, S. Marchesan and M. Prato, Angew. Chem. Int. Ed., 2015, 54, 1073410750.

26 P. Kovaříček, Z. Bastl, V. Valeš and M. Kalbáč, Chem. - Eur. J., 2016, 22, 5404-5408.

27 S. Y. Son, Y. Kim, J. Lee, G.-Y. Lee, W.-T. Park, Y.-Y. Noh, C. E. Park and T. Park, J. Am. Chem. Soc., 2016, 138, 8096-8103.

28 B. Wu, Y. Zhao, H. Nan, Z. Yang, Y. Zhang, H. Zhao, D. He, Z. Jiang, X. Liu, Y. Li, Y. Shi, Z. Ni, J. Wang, J.-B. Xu and X. Wang, Nano Lett., 2016, 16, 3754-3759.

29 A. Ciesielski, M. El Garah, S. Haar, P. Kovaříček, J.-M. Lehn and P. Samorì, Nat. Chem., 2014, 6, 1017-1023.

30 J.-M. Lehn, Prog. Polym. Sci., 2005, 30, 814-831.

31 N. Roy, B. Bruchmann and J.-M. Lehn, Chem. Soc. Rev., 2015, 44, 3786-3807.

32 A. Ciesielski, S. Lena, S. Masiero, G. P. Spada and P. Samorì, Angew. Chem. Int. Ed., 2010, 49, 1963-1966.

33 R. J. Wojtecki, M. A. Meador and S. J. Rowan, Nat. Mater., 2011, 10, 14-27.

34 K.-S. Lee, J. C. Won and J. C. Jung, Makromol. Chem., 1989, 190, 1547-1552.

35 J. Plas, D. Waghray, J. Adisoejoso, O. Ivasenko, W. Dehaen and S. D. Feyter, Chem. Commun., 2015, 51, 16338-16341.

36 P. Kovaříček and J.-M. Lehn, J. Am. Chem. Soc., 2012, 134, 9446-9455. 
37 S. Kulchat, M. N. Chaur and J.-M. Lehn, Chem. - Eur. J., 2017, 23, 11108-11118.

38 M. E. Belowich and J. Fraser. Stoddart, Chem. Soc. Rev., 2012, 41, 2003-2024.

39 Y. Yu, J. Lin and S. Lei, RSC Adv., 2017, 7, 11496-11502.

40 I. Janica, V. Patroniak, P. Samorì and A. Ciesielski, Chem. - Asian J., 2018, 13, 465-481.

41 Y. Yu, J. Sun and S. Lei, J. Phys. Chem. C, 2015, 119, 16777-16784.

42 D. F. Perepichka and F. Rosei, Science, 2009, 323, 216-217.

43 R. Gutzler and D. F. Perepichka, J. Am. Chem. Soc., 2013, 135, 16585-16594.

44 L. Xu, X. Zhou, Y. Yu, W. Q. Tian, J. Ma and S. Lei, ACS Nano, 2013, 7, 8066-8073.

45 S.-Y. Ding, J. Gao, Q. Wang, Y. Zhang, W.-G. Song, C.-Y. Su and W. Wang, J. Am. Chem. Soc., 2011,

133, 19816-19822.

46 D. N. Bunck and W. R. Dichtel, J. Am. Chem. Soc., 2013, 135, 14952-14955.

47 A. P. Côté, A. I. Benin, N. W. Ockwig, M. O’Keeffe, A. J. Matzger and O. M. Yaghi, Science, 2005, 310, 1166-1170.

48 G. Li, W.-H. Chang and Y. Yang, Nat. Rev. Mater., 2017, 2, 17043.

49 G. Feng, J. Li, F. J. M. Colberts, M. Li, J. Zhang, F. Yang, Y. Jin, F. Zhang, R. A. J. Janssen, C. Li and W.

Li, J. Am. Chem. Soc., 2017, 139, 18647-18656.

50 A. Facchetti, Chem. Mater., 2011, 23, 733-758.

51 M. Kertesz, C. H. Choi and S. Yang, Chem. Rev., 2005, 105, 3448-3481.

52 M. Ciaccia, R. Cacciapaglia, P. Mencarelli, L. Mandolini and S. Di Stefano, Chem. Sci., 2013, 4, 2253-2261.

53 J. E. Lee, G. Ahn, J. Shim, Y. S. Lee and S. Ryu, Nat. Commun., 2012, 3, 1024.

54 M. Kalbac, O. Frank and L. Kavan, Carbon, 2012, 50, 3682-3687.

55 T. Hallam, N. C. Berner, C. Yim and G. S. Duesberg, Adv. Mater. Interfaces, 2014, 1, 1400115.

56 V. Valeš, P. Kovaříček, X. Ji, X. Ling, J. Kong, M. S. Dresselhaus and M. Kalbáč, Phys. Status Solidi B, 2016, 253, 2347-2350.

$57 \mathrm{G}$. Socrates, Infrared and Raman characteristic group frequencies: tables and charts, Wiley,

Chichester, 3. ed., repr. as paperback., 2010.

58 G. R. Strobl and W. Hagedorn, J. Polym. Sci. Polym. Phys. Ed., 1978, 16, 1181-1193.

59 S. Raudenkolb, S. Wartewig and R. H. H. Neubert, Chem. Phys. Lipids, 2003, 124, 89-101.

60 L. Piot, D. Bonifazi and P. Samorì, Adv. Funct. Mater., 2007, 17, 3689-3693.

61 C.-A. Palma and P. Samorì, Nat. Chem., 2011, 3, 431-436.

62 J. A. A. W. Elemans, S. Lei and S. De Feyter, Angew. Chem. Int. Ed., 2008, 48, 7298-7332.

63 Y. Xue and M. B. Zimmt, J. Am. Chem. Soc., 2012, 134, 4513-4516.

64 A. Ciesielski, C.-A. Palma, M. Bonini and P. Samorì, Adv. Mater., 2010, 22, 3506-3520.

65 R. Zacharia, H. Ulbricht and T. Hertel, Phys. Rev. B, 2004, 69, 155406.

66 A. J. Gellman and K. R. Paserba, J. Phys. Chem. B, 2002, 106, 13231-13241.

67 Y. Okawa and M. Aono, Nature, 2001, 409, 683-684.

68 T. Takami, H. Ozaki, M. Kasuga, T. Tsuchiya, A. Ogawa, Y. Mazaki, D. Fukushi, M. Uda and M. Aono,

Angew. Chem. Int. Ed. Engl., 1997, 36, 2755-2757.

69 A. Miura, S. De Feyter, M. M. S. Abdel-Mottaleb, A. Gesquière, P. C. M. Grim, G. Moessner, M.

Sieffert, M. Klapper, K. Müllen and F. C. De Schryver, Langmuir, 2003, 19, 6474-6482.

70 Y. Yang and M. B. Zimmt, Langmuir, 2015, 31, 12408-12416.

71 T. Verhagen, B. Pacakova, M. Kalbac and J. Vejpravova, Nanomaterials, 2019, 9, 353.

72 J. J. Armao and J.-M. Lehn, Angew. Chem. Int. Ed., 2016, 55, 13450-13454.

73 J. Holub, G. Vantomme and J.-M. Lehn, J. Am. Chem. Soc., 2016, 138, 11783-11791.

74 G. Men and J.-M. Lehn, Chem. Sci., 2018, 10, 90-98.

75 S. Barik and W. G. Skene, Polym. Chem., 2011, 2, 1091-1097.

76 S. Barik, T. Bletzacker and W. G. Skene, Macromolecules, 2012, 45, 1165-1173. 
TOC graphics:

View Article Online

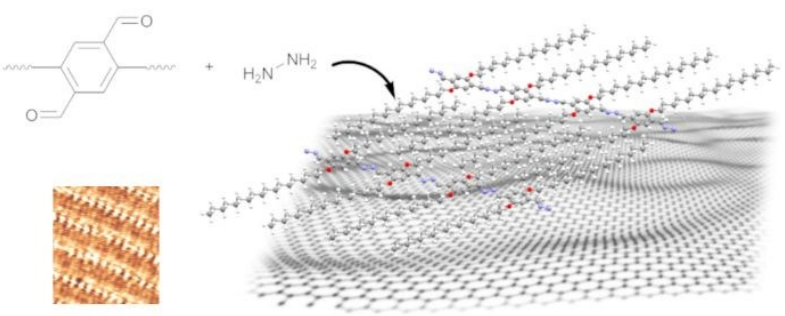

Covalently dynamic polymers self-assemble on graphene crystalline epitaxial layers to for 2-D heterostructures 